ORIGINAL

\title{
Gamma-glutamyl transferase is associated with sarcopenia and sarcopenic obesity in community-dwelling older adults: results from the Fifth Korea National Health and Nutrition Examination Survey, 2010-2011
}

\author{
Namki Hong, Eun Young Lee and Chang Oh Kim \\ Department of Internal Medicine, Yonsei University College of Medicine, Seoul, Korea
}

\begin{abstract}
Although elevated serum gamma-glutamyl transferase activity (GGT) has been linked with metabolic risk factors for sarcopenia, including non-alcoholic fatty liver disease, adiposity, and insulin resistance, whether GGT independently associated with sarcopenia and sarcopenic obesity has not yet been investigated. We analyzed crosssectional data of 3,193 community-dwelling adults $(42.2 \%$ men, age $63.4 \pm 8.7)$ aged $\geq 50$ years from the Fifth Korean National Health and Nutrition Examination Survey, 2010-2011. Sarcopenia was defined as a calculated value of the appendicular skeletal muscle mass divided by body weight $(\mathrm{ASM} / \mathrm{Wt}, \%)<1$ standard deviation below the sex-specific mean for healthy young adults. Sarcopenic obesity was defined as sarcopenia combined with a waist circumference $\geq 90$ $\mathrm{cm}$ for men and $\geq 85 \mathrm{~cm}$ for women. The prevalence of sarcopenia and sarcopenic obesity increased stepwise from the lowest to highest GGT quintiles (sarcopenia, 20.2-39.7\%; sarcopenic obesity, 7.5-27.3\%; $P$ for trend, <0.001). Serum GGT activity was associated negatively with ASM and positively with waist circumference. In multivariate logistic regression analyses, participants in the highest GGT quintile had a 2.3-fold increased risk of sarcopenia and 3.4-fold risk of sarcopenic obesity versus those in the lowest quintile, whereas each single-unit increase in natural log-GGT associated independently with a $35 \%$ increased risk of sarcopenia and $62 \%$ increased risk of sarcopenic obesity after adjusting for age, sex, body mass index, and other confounders. Elevated serum GGT activity was independently associated with sarcopenia and sarcopenic obesity in community-dwelling older adults.
\end{abstract}

Key words: Gamma-glutamyl transferase, Sarcopenia, Obesity, Insulin resistance

SARCOPENIA, a syndrome characterized by the progressive loss of skeletal muscle mass and strength with advancing age, is associated with functional limitations, morbidity, and mortality [1-4]. Sarcopenia is often concurrent with obesity, particularly the accumulation of visceral fat; this is related to chronic inflammation, increased insulin resistance, and a vicious cycle of further reductions in the skeletal muscle mass, which may synergistically promote functional and metabolic impairments [5-7]. According to previous reports, the prevalence of sarcopenia and sarcopenic obesity were relatively high and increased with age,

Submitted Feb. 24, 2015; Accepted Apr. 3, 2015 as EJ15-0119

Released online in J-STAGE as advance publication Apr. 25, 2015

Correspondence to: Chang Oh Kim, M.D., Ph.D., Division of Geriatrics, Department of Internal Medicine, Yonsei University College of Medicine, 50-1 Yonsei-ro, Seodaemun-gu, Seoul 120752, Republic of Korea. E-mail: cokim@yuhs.ac although the prevalence varied depending on definitions and reference populations $[8,9]$. Given the trend toward a rapidly aging global population, the development of effective diagnostic strategies and interventions for sarcopenia and sarcopenic obesity, including identification of biomarkers, would have health-related and socioeconomic impacts on older adults [10-12].

Serum gamma-glutamyl transferase (GGT) activity has been strongly linked with the risk of non-alcoholic fatty liver disease (NAFLD), type 2 diabetes mellitus (DM), and metabolic syndrome [13-16]. These metabolic risk factors are also known to be associated with sarcopenia, with or without visceral obesity, and are mediated by a shared pathophysiology such as insulin resistance or chronic inflammation $[5-7,17,18]$. Thus, increased serum GGT activity might indicate the presence of sarcopenia and sarcopenic obesity. However, the direct association of GGT with sarcopenia and sar- 
copenic obesity has not yet been investigated. In this context, we aimed to study whether GGT is independently associated with sarcopenia and sarcopenic obesity in community-dwelling older adults.

\section{Materials and Methods}

\section{Study sample}

We analyzed data acquired in the first and second years (2010-2011) of the Fifth Korean National Health and Nutritional Examination Survey (KNHANES), a cross-sectional national representative survey. Study candidates were selected by household units using a stratified, multistage probability sampling method. Of the 10,938 candidates in 2009 and 10,589 in 2010, $8,958(81.9 \%)$ and $8,518(80.4 \%)$, respectively, participated in the study and provided informed consent. Among 13,165 responders aged $\geq 20$ years, body composition data as assessed via whole body dual-energy x-ray absorptiometry scanning (DXA) were available for 8,204 individuals. Among them, 4,223 participants were aged $\geq 50$ years. After excluding 493 individuals with fasting times $<12$ hours, 402 individuals with any missing data, and 135 individuals with hepatitis B surface antigen positivity or liver cirrhosis, 1,348 men and 1,845 women were included in the final analysis. KNHANES was approved by the Institutional Review Board of the Korea Centers for Disease Control and Prevention (IRB No. 2010-02CON-21-C, 2011-02CON-06-C).

\section{Definitions of sarcopenia and sarcopenic obesity}

The appendicular skeletal muscle mass (ASM) was measured using DXA (Hologic Discovery-W fan-beam densitometer, Hologic, Inc., Bedford, MA, USA). ASM was calculated as the sum of the non-bone, nonfat soft tissue masses of the arms and legs. Sarcopenia was defined as an ASM value as a percentage of body weight $(\mathrm{ASM} / \mathrm{Wt} \times 100)<1$ standard deviation below the sex-specific mean for a young reference group; this was modified from the definition used by Janssen et al [2]. Among 2,477 participants of KNHANES 20102011 with DXA data who were aged 20-39 years, 323 with any self-reported history of diabetes mellitus, coronary artery disease, stroke, chronic kidney disease, tuberculosis, asthma, chronic obstructive pulmonary disease, thyroid disorder, arthritis, liver cirrhosis, and any type of cancer were excluded to define the healthy reference group. In this reference group, $\mathrm{ASM} / \mathrm{Wt}$ value (mean \pm standard deviation, $\%$ ) were as follows: $32.6 \pm 2.9$ in men and $25.8 \pm 2.4$ in women. The ASM/ Wt cutoff value for sarcopenia was $29.7 \%$ in men and $23.4 \%$ in women. Obesity was defined as a waist circumference $\geq 90 \mathrm{~cm}$ for men and $\geq 85 \mathrm{~cm}$ for women in accordance with the recommendation of the Korean Society for the Study of Obesity [19]. Sarcopenic obesity was defined as a combination of sarcopenia and obesity as defined above.

\section{Laboratory measurements}

Blood samples were sent to a certified central laboratory to determine the GGT, high-density lipoprotein cholesterol (HDL-C), triglyceride (TG), fasting blood glucose, alanine aminotransferase (ALT), aspartate aminotransferase (AST), and alkaline phosphatase (ALP) levels using a Hitachi Automatic Analyzer 7600 (Hitachi Ltd., Tokyo, Japan). The cutoff value for the normal GGT range was $<73 \mathrm{IU} / \mathrm{L}$ in men $(1,137$, $84.3 \%)$ and $<48 \mathrm{IU} / \mathrm{L}$ in women $(1,714,92.9 \%)$ [20]. The coefficient of variation for GGT was consistently $<5 \%$. The serum insulin level was measured using an immunoradiometric assay (available in 2,179 participants, 68.2\%). The homeostasis model assessment of insulin resistance (HOMA-IR) was calculated as the fasting insulin level $(\mu \mathrm{U} / \mathrm{mL}) \times$ fasting plasma glucose level $(\mathrm{mmol} / \mathrm{L}) / 22.5$ [21]. The 25-hydroxyvitamin D level was measured using a radioimmunoassay method with the 1470 WIZARD gamma-counter (PerkinElmer, Turku, Finland).

\section{Covariates}

Body mass index (BMI) was calculated as the body weight $(\mathrm{kg}) /$ height squared $\left(\mathrm{m}^{2}\right)$. WC was measured at the mid-point between the lower rib margin and upper lateral border of the ilium at the end of expiration. Total body fat mass $(\mathrm{kg})$ and percent body fat (total body fat mass/weight $\times 100, \%$ ) were measured using DXA as described above. Alcohol consumption as assessed by the self-reported drinking behavior during the previous 12 months was converted into the amount of pure alcohol consumed per day and subsequently categorized into 5 groups: none, very light $(0.1-5.0$ $\mathrm{g} /$ day), light (5.1-15.0 g/day), moderate (15.1-30.0 $\mathrm{g} /$ day), and heavy ( $>30 \mathrm{~g} /$ day) [22]. Physical activities were expressed as metabolic equivalents and were grouped as high, moderate, and low according to the International Physical Activity Questionnaire [23]. Diabetes mellitus was defined as the composite pres- 
ence of self-reported doctor-diagnosed diabetes mellitus or a fasting glucose level $\geq 126 \mathrm{mg} / \mathrm{dL}$. The selfreported smoking status, education level, and presence of doctor-diagnosed chronic conditions were recorded.

\section{Statistical analyses}

Data are presented as means \pm standard deviations, medians [interquartile ranges] or numbers (\%). Comparisons of participant characteristics were performed using the independent $t$-test or Wilcoxon ranksum test for continuous variables and the chi-square test for categorical variables as appropriate. Factors correlated with GGT, including ASM, WC, sex, alcohol consumption, TG, and other covariates, were assessed in univariate and multivariate linear regression analyses with comparisons of the standardized partial regression coefficients. Independent associations of GGT with sarcopenia and sarcopenic obesity were assessed using multivariate logistic regression analyses. GGT was entered into the model in the form of either natural logged GGT (log-GGT) as a continuous variable or GGT quintiles as a categorical variable. Models were adjusted for potent covariates, including age; sex; BMI; physical activity; smoking status; alcohol consumption; education level; occupation; presence of DM, hypertension, coronary heart disease, chronic kidney disease, stroke, or any type of cancer; and TG, HDL-C, ALT, and 25(OH)D levels. A 2-sided $P<0.05$ was considered significant. All statistical analyses were performed using STATA 12.1 (StataCorp LP, College Station, TX, USA).

\section{Results}

\section{Characteristics of study participants}

The mean age of the study participants was $63.4 \pm$ 8.7 years; $1,348(42.2 \%)$ were men. Sarcopenia was present in 369 men (27.4\%) and 649 women (35.2\%). Among them, 188 men (13.9\%) and 371 women (20.1\%) had concurrent obesity. The median GGT level was $22 \mathrm{IU} / \mathrm{L}$ (interquartile range, 16-36) and most participants (89.3\%) had GGT values within the normal range. Compared to participants without sarcopenia, the serum GGT level was significantly higher in those with sarcopenia (33 [22-57] vs. 29 [20-52] in men, $P<0.001 ; 20$ [15-30] vs. 17 [13-25] in women, $P<0.001)$ or sarcopenic obesity (39 [26-65] vs. 29 [20-52] in men, $P<0.001 ; 22$ [16-33] vs. 17 [13-25] in women, $P<0.001$; Table 1). Participants with sar- copenia were older, more often obese, and had lower HDL-C, higher TG, ALT, and higher HOMA-IR levels than participants without sarcopenia. The former group was also more likely to have a sedentary occupation and less likely to exercise regularly. The serum AST and ALP levels and average amount of alcohol consumption did not differ significantly between participants with or without sarcopenia. Similar patterns were observed in participants with sarcopenic obesity.

\section{Associations between GGT and body composition parameters}

Associations between GGT and body composition parameters were assessed using univariate and multivariate linear regression analyses with GGT as a dependent variable (Table 2). Serum GGT activity was associated positively with WC but negatively with ASM after adjusting for age, sex, BMI, and other confounders. When the standardized partial regression coefficients (St- $\beta$ ) were compared, the absolute St $\beta$ value for ASM (St- $\beta=-0.18, P<0.001)$ was approximately 2 -fold higher than that for WC (St- $\beta=0.09, P<0.001)$, and ASM was a relatively strong determinant of GGT after ALT (St- $\beta=0.42, P<0.001)$, sex (St- $\beta=-0.28, P$ $<0.001$ ), and heavy alcohol consumption (St $\beta=0.21$, $P<0.001)$. When total body fat mass entered into multivariate model instead of WC, the independent association of ASM with GGT was consistent (St- $\beta=-0.14$, $P<0.001)$ whereas total body fat mass showed marginal statistical significance (St $\beta=0.06, P=0.051$ ). $25(\mathrm{OH}) \mathrm{D}$ was not an independent predictor of serum GGT activity in multivariate model $(\beta=-0.002, P=$ $0.108)$.

\section{Independent association of serum GGT level with sarcopenia/sarcopenic obesity}

The prevalence of sarcopenia and sarcopenic obesity increased in a stepwise fashion as the GGT quintile increased (for sarcopenia, $20.2 \%$ in the lowest GGT quintile [Q1] to $40.0 \%$ in the highest GGT quintile [Q5], P for trend $<0.001$; for sarcopenic obesity, $7.5 \%$ in GGT Q1 to $27.4 \%$ in GGT Q5, P for trend $<0.001$; Fig. 1). The independent associations of GGT with sarcopenia and sarcopenic obesity were assessed using multivariate logistic regression analyses (Fig. 2). The stepwise increase in the risk of sarcopenia according to GGT quintile was observed in the univariate analysis (GGT Q2: odds ratio [OR] $=1.56,95 \%$ confidence interval [CI] 1.18-2.05, $P=0.002$; GGT Q5: OR 
Table 1 Comparison of study participant characteristics by the presence of sarcopenia and sarcopenic obesity

\begin{tabular}{|c|c|c|c|c|c|c|}
\hline & \multicolumn{3}{|c|}{$\begin{array}{c}\text { Men } \\
(\mathrm{N}=1,348)\end{array}$} & \multicolumn{3}{|c|}{$\begin{array}{c}\text { Women } \\
(\mathrm{N}=1,845)\end{array}$} \\
\hline & $\begin{array}{c}\text { No sarcopenia } \\
(\mathrm{N}=979)\end{array}$ & $\begin{array}{l}\text { Sarcopenia } \\
(\mathrm{N}=369)\end{array}$ & $\begin{array}{c}\text { Sarcopenic obesity } \\
(\mathrm{N}=188)\end{array}$ & $\begin{array}{l}\text { No sarcopenia } \\
(\mathrm{N}=1,196\end{array}$ & $\begin{array}{l}\text { Sarcopenia } \\
(\mathrm{N}=649)\end{array}$ & $\begin{array}{c}\text { Sarcopenic obesity } \\
(\mathrm{N}=371)\end{array}$ \\
\hline Age, years & $63.1 \pm 8.4$ & $65.6 \pm 8.3^{*}$ & $65.6 \pm 7.9^{*}$ & $62.8 \pm 9.1$ & $63.7 \pm 8.7^{*}$ & $64.9 \pm 8.4^{*}$ \\
\hline BMI, $\mathrm{kg} / \mathrm{m}^{2}$ & $23.2 \pm 2.7$ & $25.2 \pm 2.6^{*}$ & $26.7 \pm 2.2^{*}$ & $23.2 \pm 2.8$ & $26.0 \pm 3.4^{*}$ & $27.8 \pm 2.9^{*}$ \\
\hline $\mathrm{WC}, \mathrm{cm}$ & $83.8 \pm 8.2$ & $90.1 \pm 7.3^{*}$ & $95.6 \pm 4.9^{*}$ & $79.8 \pm 8.3$ & $86.6 \pm 9.2^{*}$ & $92.9 \pm 6.2 *$ \\
\hline ASM, kg & $21.0 \pm 2.8$ & $19.4 \pm 2.4^{*}$ & $20.7 \pm 2.2$ & $14.2 \pm 1.9$ & $13.3 \pm 1.9^{*}$ & $14.2 \pm 1.8$ \\
\hline $\mathrm{ASM} / \mathrm{Wt}, \%$ & $32.6 \pm 1.9$ & $28.0 \pm 1.4^{*}$ & $27.7 \pm 1.4^{*}$ & $25.9 \pm 1.9$ & $21.9 \pm 1.3^{*}$ & $21.6 \pm 1.4^{*}$ \\
\hline ASM/Height ${ }^{2}, \mathrm{~kg} / \mathrm{m}^{2}$ & $7.5 \pm 0.8$ & $7.1 \pm 0.7^{*}$ & $7.4 \pm 0.6$ & $6.0 \pm 0.6$ & $5.6 \pm 0.7^{*}$ & $6.0 \pm 0.6$ \\
\hline Total body fat mass, $\mathrm{kg}$ & $12.7 \pm 3.8$ & $18.7 \pm 3.8^{*}$ & $20.7 \pm 3.4^{*}$ & $17.1 \pm 4.3$ & $23.4 \pm 4.9^{*}$ & $25.8 \pm 4.6^{*}$ \\
\hline Percent body fat, $\%$ & $21.2 \pm 4.4$ & $29.2 \pm 3.4^{*}$ & $30.3 \pm 3.2 *$ & $33.4 \pm 4.7$ & $41.6 \pm 3.5^{*}$ & $42.5 \pm 3.3^{*}$ \\
\hline Systolic BP, mmHg & $125 \pm 16$ & $127 \pm 16$ & $128 \pm 15^{*}$ & $125 \pm 18$ & $129 \pm 16^{*}$ & $132 \pm 16^{*}$ \\
\hline GGT, IU/L & $29[20-52]$ & $33[22-57]^{*}$ & $39[26-65]^{*}$ & 17 [13-25] & $20[15-30]^{*}$ & $22[16-33]^{*}$ \\
\hline ALT, IU/L & $20[15-27)$ & $21[16-31)^{*}$ & $23[17-35]^{*}$ & $16[13-21]$ & $18[14-23]^{*}$ & $19[15-25]^{*}$ \\
\hline AST, IU/L & 22 [19-27] & 23 [20-29] & $24[20-30]^{*}$ & $21[18-24]$ & $21[18-24]$ & $21[18-25]$ \\
\hline ALP, IU/L & 232 [195-276] & $230[194-271]$ & 223 [193-266] & $240[200-290]$ & 246 [201-294] & 247 [201-295] \\
\hline $\mathrm{HDL}-\mathrm{C}, \mathrm{mg} / \mathrm{dL}$ & $46.7 \pm 11.6$ & $42.0 \pm 10.1^{*}$ & $41.2 \pm 9.6^{*}$ & $49.4 \pm 11.3$ & $47.5 \pm 10.3 *$ & $46.0 \pm 9.9^{*}$ \\
\hline $\mathrm{TG}, \mathrm{mg} / \mathrm{dL}$ & $154 \pm 148$ & $178 \pm 143^{*}$ & $192 \pm 161^{*}$ & $130 \pm 80$ & $143 \pm 75^{*}$ & $152 \pm 78^{*}$ \\
\hline Fasting glucose, mg/dL & $103 \pm 26$ & $109 \pm 25^{*}$ & $111 \pm 26^{*}$ & $99 \pm 22$ & $101 \pm 22^{*}$ & $105 \pm 26^{*}$ \\
\hline HOMA-IR $(\mathrm{N}=2,271)$ & $2.1[1.7-2.8]$ & $2.7 *[2.0-3.4]$ & $3.0 *[2.4-3.7]$ & $2.1[1.7-2.8]$ & $2.5 *[1.9-3.3]$ & $2.9^{*}[2.1-3.7]$ \\
\hline $25(\mathrm{OH}) \mathrm{D}, \mathrm{ng} / \mathrm{mL}$ & $20.3 \pm 7.1$ & $18.8 \pm 6.3^{*}$ & $18.8 \pm 6.2^{*}$ & $18.0 \pm 6.9$ & $16.9 \pm 6.8^{*}$ & $16.6 \pm 6.7^{*}$ \\
\hline \multicolumn{7}{|l|}{ Alcohol consumption } \\
\hline Very light $(0-5 \mathrm{~g} / \mathrm{d})$ & $466(47.6)$ & $200(54.4)$ & $100(53.3)$ & $1109(92.9)$ & $607(93.6)$ & $347(93.6)$ \\
\hline Light $(5.1-15 \mathrm{~g} / \mathrm{d})$ & $134(13.7)$ & $49(13.1)$ & $24(12.7)$ & $45(3.6)$ & $23(3.5)$ & $15(4.0)$ \\
\hline Moderate $(15.1-30 \mathrm{~g} / \mathrm{d})$ & $184(18.8)$ & $54(14.6)$ & $25(13.3)$ & $33(2.8)$ & $15(2.3)$ & $6(1.6)$ \\
\hline Heavy $(>30 \mathrm{~g} / \mathrm{d})$ & 195 (19.9) & $66(17.9)$ & $39(20.7)$ & $9(0.7)$ & $4(0.6)$ & $3(0.8)$ \\
\hline \multicolumn{7}{|l|}{ Physical activity } \\
\hline Low & $391(39.9)$ & $162(43.9)^{*}$ & $82(43.6)^{*}$ & $569(47.6)$ & 337 (51.9) & $196(52.8)$ \\
\hline Moderate & $295(30.1)$ & $135(36.6)^{*}$ & $72(38.3)^{*}$ & $380(31.7)$ & 205 (31.6) & $112(30.2)$ \\
\hline High & $293(30.0)$ & $78(19.5)^{*}$ & $34(18.1)^{*}$ & 247 (20.7) & $107(16.5)$ & $63(17.0)$ \\
\hline \multicolumn{7}{|l|}{ Education } \\
\hline Primary school & $333(34.0)$ & $112(30.3)$ & $54(28.7)$ & $722(60.3)$ & 395 (60.9) & $254(68.4)^{*}$ \\
\hline Middle school & $196(20.0)$ & $84(22.8)$ & 45 (23.9) & $192(16.1)$ & $108(16.6)$ & $57(15.4)^{*}$ \\
\hline$\geq$ High school & $450(46.0)$ & $183(46.9)$ & $89(47.4)$ & $282(23.6)$ & $146(22.5)$ & $60(16.2)^{*}$ \\
\hline \multicolumn{7}{|l|}{ Occupation } \\
\hline Unemployed & $291(29.7)$ & $167(45.2)^{*}$ & $82(43.6)^{*}$ & $684(57.2)$ & $411(63.3)^{*}$ & $237(63.8)^{*}$ \\
\hline Physical work & 499 (50.9) & $116(31.4)^{*}$ & $64(34.0)^{*}$ & $333(27.8)$ & $144(22.2)^{*}$ & $83(22.4)^{*}$ \\
\hline Sedentary work & $189(19.4)$ & $86(23.4)^{*}$ & $42(22.4)^{*}$ & $179(15.0)$ & $94(14.5)^{*}$ & $51(13.8)^{*}$ \\
\hline \multicolumn{7}{|l|}{ Smoking status } \\
\hline Never & $152(15.5)$ & $55(14.9)^{*}$ & $30(15.9)$ & 1098(91.8) & $614(94.6)^{*}$ & $350(94.4)^{*}$ \\
\hline Ex-smoker & $496(50.6)$ & $225(60.9)^{*}$ & $108(57.5)$ & $42(3.5)$ & $20(3.1)^{*}$ & $15(4.0)^{*}$ \\
\hline Current & 331 (33.9) & $89(24.2)^{*}$ & $50(26.6)$ & $56(4.7)$ & $15(2.3)^{*}$ & $6(1.6)^{*}$ \\
\hline DM & $160(16.3)$ & $106(28.7)^{*}$ & $58(30.8)^{*}$ & $161(13.5)$ & $117(18.0)^{*}$ & $87(23.4)^{*}$ \\
\hline Hypertension & $312(31.8)$ & $199(53.9)^{*}$ & $109(57.9)^{*}$ & $431(36.0)$ & $331(51.0)^{*}$ & $231(59.7)^{*}$ \\
\hline Stroke & $30(3.1)$ & $26(7.1)^{*}$ & $17(9.0)^{*}$ & $33(2.8)$ & $21(3.2)$ & $15(4.0)$ \\
\hline Coronary heart disease & $41(4.2)$ & $41(11.1)^{*}$ & $19(10.1)^{*}$ & $50(4.2)$ & $35(5.4)$ & $23(6.2)$ \\
\hline Chronic kidney disease & $50(5.1)$ & $53(14.4)^{*}$ & $28(14.9)^{*}$ & $55(4.6)$ & $36(5.5)$ & $31(8.4)^{*}$ \\
\hline Any cancer & $41(4.2)$ & $23(6.2)$ & $11(5.8)$ & $68(5.7)$ & $32(4.9)$ & $18(4.8)$ \\
\hline
\end{tabular}

*: $P<0.05$ when compared with the no sarcopenia group as a reference in each sex strata.

Continuous variables are presented as means \pm standard deviations or medians [interquartile ranges] and categorical variables are presented as numbers (\%). BMI, body mass index; WC, waist circumference; ASM, appendicular skeletal muscle mass; Wt, body weight; GGT, gamma-glutamyl transferase; ALT, alanine aminotransferase; AST, aspartate aminotransferase; ALP, alkaline phosphatase; HDL-C, high-density lipoprotein cholesterol; TG, triglyceride; HOMA-IR, homeostatic model assessment of insulin resistance; 25(OH) D, serum 25-hydroxyvitamin D concentration; DM, diabetes mellitus. 
Table 2 Associations of body composition parameters with gamma-glutamyl transferase and other covariates in the univariate and multivariate linear regression analyses

\begin{tabular}{|c|c|c|c|c|c|}
\hline & \multicolumn{2}{|c|}{ Univariate } & \multicolumn{2}{|c|}{ Multivariate } & \multirow[b]{2}{*}{$P$-value } \\
\hline & $\beta$ (S.E) & $P$-value & $\beta$ (S.E) & Standardized $\beta$ & \\
\hline ASM (kg) & $0.061(0.003)$ & $<0.001$ & $-0.032(0.005)$ & -0.18 & $<0.001$ \\
\hline $\mathrm{WC}(\mathrm{cm})$ & $0.021(0.001)$ & $<0.001$ & $0.007(0.002)$ & 0.09 & $<0.001$ \\
\hline BMI $\left(\mathrm{kg} / \mathrm{m}^{2}\right)$ & $0.035(0.003)$ & $<0.001$ & $-0.002(0.006)$ & -0.01 & 0.768 \\
\hline Age (years) & $-0.006(0.001)$ & $<0.001$ & $-0.002(0.001)$ & -0.03 & 0.049 \\
\hline Women & $-0.557(0.022)$ & $<0.001$ & $-0.397(0.046)$ & -0.28 & $<0.001$ \\
\hline Moderate alcohol* & $0.514(0.041)$ & $<0.001$ & $0.390(0.039)$ & 0.15 & $<0.001$ \\
\hline Heavy alcohol* & $0.863(0.041)$ & $<0.001$ & $0.540(0.042)$ & 0.21 & $<0.001$ \\
\hline Current smoker & $0.513(0.032)$ & $<0.001$ & $0.235(0.032)$ & 0.11 & $<0.001$ \\
\hline HTN & $0.102(0.024)$ & $<0.001$ & $0.049(0.022)$ & 0.03 & 0.030 \\
\hline $\mathrm{TG}(\mathrm{mg} / \mathrm{dL})$ & $0.002(0.001)$ & $<0.001$ & $0.002(0.001)$ & 0.15 & $<0.001$ \\
\hline HOMA-IR & $0.086(0.009)$ & $<0.001$ & $0.021(0.007)$ & 0.05 & 0.006 \\
\hline Log-ALT (IU/L) & $0.812(0.022)$ & $<0.001$ & $0.660(0.025)$ & 0.42 & $<0.001$ \\
\hline
\end{tabular}

* Compared with non-drinkers. ASM, appendicular skeletal muscle mass; WC, waist circumference; BMI, body mass index; HTN, hypertension; TG, triglyceride; HOMA-IR, homeostatic model assessment of insulin resistance; 25(OH)D, serum 25-hydroxyvitamin D concentration; ALT, alanine aminotransferase.
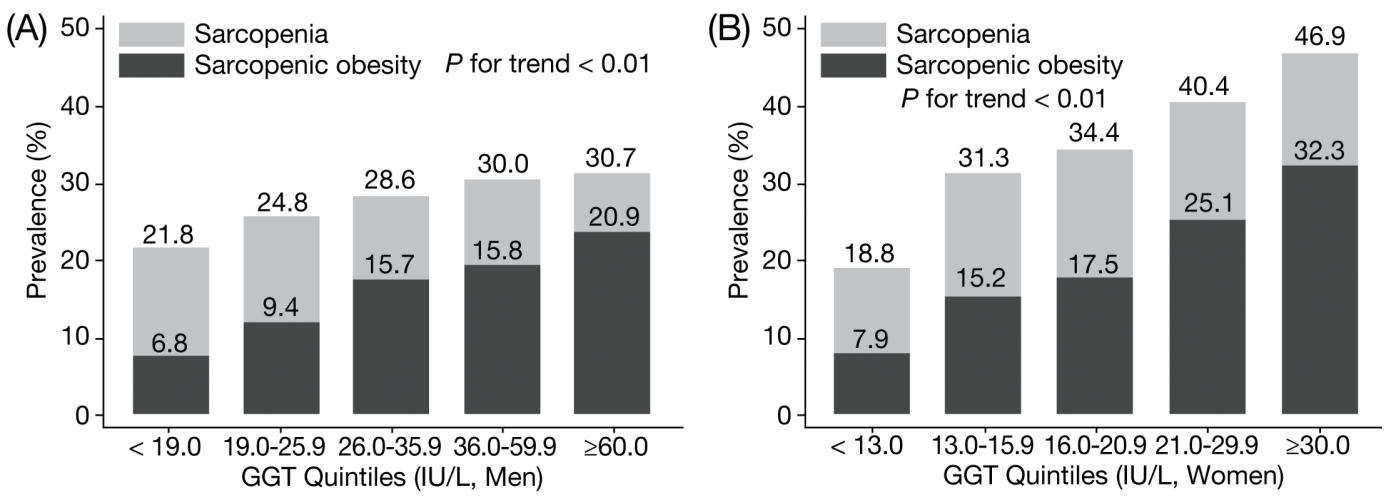

Fig. 1 Prevalence of sarcopenia and sarcopenic obesity according to sex-specific gamma-glutamyl transferase (GGT) quintiles

\begin{tabular}{|c|c|c|c|c|}
\hline Subgroups & Prevalence (N, \%) & & $\begin{array}{l}\text { Odds } \\
\text { ratio }(95 \% \mathrm{Cl})\end{array}$ & $P$-value \\
\hline \multicolumn{5}{|l|}{ Sarcopenia } \\
\hline Overall & 1018/3193, 31.9\% & $=$ & $1.35(1.15-1.58)$ & $<0.001$ \\
\hline GGT Q2 (vs. Q1) & & & $1.48(1.11-2.13)$ & 0.009 \\
\hline GGT Q3 (vs. Q1) & & & $1.59(1.19-2.13)$ & 0.002 \\
\hline GGT Q4 (vs. Q1) & & & $1.97(1.45-2.68)$ & $<0.001$ \\
\hline GGT Q5 (vs. Q1) & & & $2.34(1.68-3.26)$ & $<0.001$ \\
\hline Men & $369 / 1348,27.4 \%$ & $\rightarrow$ & $1.26(1.01-1.59)$ & 0.041 \\
\hline Women & $649 / 1845,35.2 \%$ & $\rightarrow$ & $1.51(1.18-1.90)$ & $<0.001$ \\
\hline Age $<65$ & $516 / 1783,28.9 \%$ & $\rightarrow$ & $1.34(1.08-1.68)$ & 0.008 \\
\hline Age $\geq 65$ & $502 / 1410,35.6 \%$ & $\rightarrow$ & $1.33(1.04-1.71)$ & 0.023 \\
\hline $\mathrm{BMl}<25$ & $450 / 2081,21.6 \%$ & $\rightarrow$ & $1.34(1.09-1.65)$ & 0.006 \\
\hline $\mathrm{BMI} \geq 25$ & $568 / 1112,51.1 \%$ & - & $1.34(1.03-1.74)$ & 0.027 \\
\hline \multicolumn{5}{|c|}{ Sarcopenic obesity } \\
\hline Overall & $559 / 3193,17.5 \%$ & & $1.62(1.30-2.01)$ & $<0.001$ \\
\hline GGT Q2 (vs. Q1) & & & $1.51(0.95-2.40)$ & 0.081 \\
\hline GGT Q3 (vs. Q1) & & & $1.70(1.09-2.65)$ & 0.019 \\
\hline GGT Q4 (vs. Q1) & & $\longrightarrow$ & $2.30(1.45-3.65)$ & $<0.001$ \\
\hline GGT Q5 (vs. Q1) & & & $3.42(2.10-5.55)$ & $<0.001$ \\
\hline Men & $188 / 1348,13.9 \%$ & $\rightarrow-$ & $1.80(1.31-2.46)$ & $<0.001$ \\
\hline Women & $371 / 1845,20.1 \%$ & $\rightarrow-$ & $1.49(1.09-2.04)$ & 0.012 \\
\hline Age $<65$ & $262 / 1783,14.7 \%$ & $\rightarrow$ & $1.57(1.16-2.14)$ & 0.003 \\
\hline Age $\geq 65$ & $297 / 1410,21.1 \%$ & $\Rightarrow$ & $1.62(1.16-2.26)$ & 0.004 \\
\hline $\mathrm{BMI}<25$ & $89 / 2081,4.3 \%$ & $\rightarrow-$ & $1.60(1.09-2.37)$ & 0.017 \\
\hline $\mathrm{BMI} \geq 25$ & $470 / 1112,42.3 \%$ & $\Rightarrow$ & $1.63(1.24-2.14)$ & $<0.001$ \\
\hline
\end{tabular}

Fig. 2 Adjusted odds ratios for presence of sarcopenia or sarcopenic obesity per single-unit increase in natural log-gamma-glutamyl transferase (GGT) as a continuous variable (presented in overall group and subgroups stratified by sex, age, and BMI) and per GGT quintile as a categorical variable using the lowest GGT quintile as a reference group (GGT Q2 - Q5 vs. Q1) by using multivariate logistic regression model. All models were adjusted for potent covariates including age; sex; BMI; physical activity; smoking status; alcohol consumption; education level; occupation; presence of diabetes, hypertension, coronary heart disease, chronic kidney disease, stroke, or any type of cancer; and triglyceride, high-density lipoprotein cholesterol, alanine aminotransferase, and 25-hydroxyvitamin D levels. 
$=2.62,95 \% \mathrm{CI}, 2.01-3.42, P<0.001)$. Similar patterns were observed for the risk of sarcopenic obesity (GGT Q2: OR $=1.79,95 \%$ CI, $1.19-2.68, P=0.005$; GGT Q5: OR $=4.66,95 \%$ CI, 3.21-6.77, $P<0.001$ ). These associations were robust after adjusting for potent covariates in multivariate model (Fig. 2). Each single-unit increase in log-GGT was associated with a $35 \%$ increased risk of sarcopenia and $62 \%$ increased risk of sarcopenic obesity among overall participants in the multivariate model. Similar results were obtained when BMI was substituted for total body fat mass (for sarcopenia: $\mathrm{OR}=1.27,95 \%$ CI 1.05-1.53, $P=0.012$; for sarcopenic obesity: $\mathrm{OR}=1.51,95 \%$ CI $1.18-1.92$, $P<0.001)$. Among other hepatic enzymes, significant associations of ALT with sarcopenia (OR $=1.33,95 \%$ CI, 1.13-1.57, $P<0.001$ ) and sarcopenic obesity (OR $=1.75,95 \%$ CI, $1.45-2.13, P<0.001)$ were observed in univariate analyses, but the significance was attenuated in the multivariate model (sarcopenia, $\mathrm{OR}=0.93$, 95\% CI, 0.75-1.16, $P=0.547$; sarcopenic obesity, OR $=0.89,95 \% \mathrm{CI}, 0.67-1.18, P=0.451)$. AST and ALP did not show any significant association with sarcopenia in either the univariate or multivariate model. In the subpopulation of participants with available HOMA-IR data $(\mathrm{N}=2,179,68.2 \%)$, the associations of GGT with sarcopenia and sarcopenic obesity remained independent after further adjusting for HOMA-IR (OR for sarcopenia $=1.24,95 \% \mathrm{CI}, 1.03-1.51, P=0.024$; OR for sarcopenic obesity $=1.55,95 \%$ CI, $1.19-2.02$, $P<0.001)$. We did not observe any effect modification of the relationships of GGT with sarcopenia and sarcopenic obesity according to age, sex, and BMI.

\section{Discussion}

The principal findings of our study are as follows: 1) the prevalence of sarcopenia and sarcopenic obesity increased stepwise across GGT quintiles in community-dwelling older adults; 2) serum GGT showed negative and positive associations with ASM and waist circumference, respectively, after adjusting for other covariates; 3 ) elevated serum GGT activity, even within the normal range, was independently associated with the presence of sarcopenia and sarcopenic obesity in the overall population and in subgroups stratified by age, sex, and BMI.

The sarcopenia cutoff value (ASM/Wt 29.7\%, men; $23.4 \%$, women) and prevalence $(27.4 \%$ for men and $35.2 \%$ for women) according to the weight-adjusted
ASM reported in this study was largely consistent with the results of previous community-based studies $[7,9]$. We used weight-adjusted ASM (ASM/Wt) to define sarcopenia based on previous studies that suggested that ASM/Wt might be a more appropriate method of defining sarcopenia and sarcopenic obesity than height-adjusted ASM (ASM/ $\mathrm{Ht}^{2}$ ) because of better correlations with insulin resistance and abdominal obesity [7,9]. Although the definitions and classifications of sarcopenia and sarcopenic obesity remain under debate, we found negative correlations of ASM/ Wt with HOMA-IR, WC, BMI, and serum TG levels whereas ASM $/ \mathrm{Ht}^{2}$ correlated positively with these factors, in accordance with previous findings. Advanced age, insulin resistance, dyslipidemia, a low physical activity level, presence of DM, hypertension, and a low serum $25(\mathrm{OH}) \mathrm{D}$ level were significantly associated with sarcopenia and sarcopenic obesity in our study, in accordance with previous studies [24]. We also found robust associations of serum GGT activity with sarcopenia and sarcopenic obesity that were independent of age, sex, BMI, and other established covariates in an elderly population. A number of studies found significant interactions of GGT and BMI with metabolic risks; particularly, the associations of higher BMI with the risk of type $2 \mathrm{DM}$, poor glycemic control, and dyslipidemia were strong in individuals with high normal serum GGT activity levels but were weak or even insignificant in individuals with low normal GGT levels $[25,26]$. Our results support the finding that higher serum GGT activity may confer an additional risk, along with BMI, upon adverse metabolic profiles by reflecting an imbalance between decreased ASM and increased visceral fat mass within an identical BMI range.

NAFLD is one of the possible links between elevated serum GGT activity and the presence of sarcopenia and sarcopenic obesity. A recent observational cohort study suggested that those with a lower muscle mass had a higher risk of NAFLD than individuals with preserved muscle mass [27]. Muscle mass loss is associated with increased visceral fat and insulin resistance; insulin resistance is known to facilitate fat accumulation in hepatocytes $[5,28]$. Pro-inflammatory cytokines and adipokines from visceral fat and ectopic fat accumulation in the liver induce chronic systemic inflammation, which lead to further losses of muscle mass and increased insulin resistance, thus forming a vicious cycle [6]. Previous studies have reported strong asso- 
ciations of GGT, a sensitive marker of NAFLD, with insulin resistance, dyslipidemia, and inflammatory cytokines $[14,15,17,28]$. In line with previous findings, HOMA-IR (insulin resistance index), ALT (more specific surrogate marker of intrahepatic TG content), and serum TG levels were all independent determinants of serum GGT activity in this study. However, we observed that the associations of GGT with sarcopenia and sarcopenic obesity were robust even after adjusting for HOMA-IR, ALT, TG, and HDL, suggesting that GGT might not be merely a simple mediator of NAFLD and insulin resistance in association with sarcopenia. Oxidative stress and mitochondrial dysfunction have previously been linked to muscle wasting and sarcopenia [29]. Given that GGT is considered as an in vivo biomarker of oxidative stress, the associations of GGT with sarcopenia and sarcopenic obesity might be partly mediated by oxidative stress $[15,17]$. Taken together, GGT might play a role as an integrative marker for sarcopenia and sarcopenic obesity, thus reflecting various predisposing conditions that are not mutually exclusive.

Our study has several limitations. The causal relationships of GGT with sarcopenia and sarcopenic obesity could not be assessed because of the cross-sectional study design. This study might not have been free from selection bias, as the data did not include the institutionalized elderly population despite using a large national representative sample. We defined sarcopenic obesity as the composite of sarcopenia and abdominal obesity measured by WC instead of BMI. Although BMI was the most popular proxy for thinness and fatness, previous studies suggested that WC as a surrogate for visceral fat area might provide better identification of metabolic impairments than other anthropometric indices in Asian population [30, 31].
The importance of muscle strength and physical performance on sarcopenia diagnosis was emphasized in current consensus reports [11]. Data regarding muscle strength or muscle quality were not available in our study, although our physical activity measurements were based on metabolic equivalents, in accordance with established guidelines [23]. However, our results might suggest that serum GGT is a sensitive indicator of sarcopenia and sarcopenic obesity in community-dwelling older individuals with or without preserved muscle strength, although further studies on the associations of GGT with muscle strength and physical performance are needed [11]. Inflammatory cytokines, adipokines, and myokines were not measured in our study. Our data lacked any direct measures of NAFLD such as imaging or liver biopsy, although we used ALT, a more specific marker of intrahepatic TG contents relative to other liver enzymes, as a surrogate marker [28, 32].

In conclusion, serum GGT activity was independently associated with the presence of sarcopenia and sarcopenic obesity in community-dwelling older people. Considering the low cost and wide availability of GGT testing in most primary care settings, measurement of serum GGT activity may facilitate the identification of individuals at a high risk of sarcopenia and sarcopenic obesity within a community. Longitudinal researches are needed to confirm the associations of GGT with sarcopenia and sarcopenic obesity in older adults.

\section{Acknowledgements}

None.

\section{Disclosure}

None declared.

\section{References}

1. Evans WJ (1995) What is sarcopenia? J Gerontol A Biol Sci Med Sci 50 Spec No: 5-8.

2. Janssen I, Heymsfield SB, Ross R (2002) Low relative skeletal muscle mass (sarcopenia) in older persons is associated with functional impairment and physical disability. J Am Geriatr Soc 50: 889-896.

3. Batsis JA, Mackenzie TA, Barre LK, Lopez-Jimenez F, Bartels SJ (2014) Sarcopenia, sarcopenic obesity and mortality in older adults: results from the National Health and Nutrition Examination Survey III. Eur $J$
Clin Nutr 68: 1001-1007.

4. Kim JH, Lim S, Choi SH, Kim KM, Yoon JW, et al. (2014) Sarcopenia: an independent predictor of mortality in community-dwelling older Korean men. $J$ Gerontol A Biol Sci Med Sci 69: 1244-1252.

5. Roubenoff R (2000) Sarcopenic obesity: does muscle loss cause fat gain? Lessons from rheumatoid arthritis and osteoarthritis. Ann N Y Acad Sci 904: 553-557.

6. Cesari M, Kritchevsky SB, Baumgartner RN, Atkinson HH, Penninx BW, et al. (2005) Sarcopenia, obe- 
sity, and inflammation--results from the Trial of Angiotensin Converting Enzyme Inhibition and Novel Cardiovascular Risk Factors study. Am J Clin Nutr 82: 428-434.

7. Lim S, Kim JH, Yoon JW, Kang SM, Choi SH, et al. (2010) Sarcopenic obesity: prevalence and association with metabolic syndrome in the Korean Longitudinal Study on Health and Aging (KLoSHA). Diabetes Care 33: 1652-1654.

8. Batsis JA, Barre LK, Mackenzie TA, Pratt SI, LopezJimenez F, et al. (2013) Variation in the prevalence of sarcopenia and sarcopenic obesity in older adults associated with different research definitions: dual-energy X-ray absorptiometry data from the National Health and Nutrition Examination Survey 1999-2004. J Am Geriatr Soc 61: 974-980.

9. Kim YS, Lee Y, Chung YS, Lee DJ, Joo NS, et al. (2012) Prevalence of sarcopenia and sarcopenic obesity in the Korean population based on the Fourth Korean National Health and Nutritional Examination Surveys. J Gerontol A Biol Sci Med Sci 67: 1107-1113.

10. Christensen K, Doblhammer G, Rau R, Vaupel JW (2009) Ageing populations: the challenges ahead. Lancet 374: 1196-1208.

11. Cruz-Jentoft AJ, Baeyens JP, Bauer JM, Boirie Y, Cederholm T, et al. (2010) Sarcopenia: European consensus on definition and diagnosis: Report of the European Working Group on Sarcopenia in Older People. Age Ageing 39: 412-423.

12. Janssen I, Shepard DS, Katzmarzyk PT, Roubenoff R (2004) The healthcare costs of sarcopenia in the United States. J Am Geriatr Soc 52: 80-85.

13. Kunutsor SK, Abbasi A, Adler AI (2014) Gammaglutamyl transferase and risk of type II diabetes: an updated systematic review and dose-response metaanalysis. Ann Epidemiol 24: 809-816.

14. Lee DS, Evans JC, Robins SJ, Wilson PW, Albano I, et al. (2007) Gamma glutamyl transferase and metabolic syndrome, cardiovascular disease, and mortality risk: the Framingham Heart Study. Arterioscler Thromb Vasc Biol 27: 127-133.

15. Whitfield JB (2001) Gamma glutamyl transferase. Crit Rev Clin Lab Sci 38: 263-355.

16. Akehi Y, Tsutsumi Y, Tatsumoto A, Yoshida R, Ohkubo $\mathrm{K}$, et al. (2010) Serum gamma-glutamyltransferase, triglyceride and total cholesterol are possible prediabetic risk markers in young Japanese men. Endocr J 57: 981989.

17. Bradley RD, Fitzpatrick AL, Jacobs DR, Jr., Lee DH, Swords Jenny N, et al. (2014) Associations between gamma-glutamyltransferase (GGT) and biomarkers of atherosclerosis: the multi-ethnic study of atherosclerosis (MESA). Atherosclerosis 233: 387-393.

18. Moon SS (2014) Low skeletal muscle mass is associ- ated with insulin resistance, diabetes, and metabolic syndrome in the Korean population: the Korea National Health and Nutrition Examination Survey (KNHANES) 2009-2010. Endocr J 61: 61-70.

19. Lee SY, Park HS, Kim DJ, Han JH, Kim SM, et al. (2007) Appropriate waist circumference cutoff points for central obesity in Korean adults. Diabetes Res Clin Pract 75: 72-80.

20. Persijn JP, van der Slik W (1976) A new method for the determination of gamma-glutamyltransferase in serum. J Clin Chem Clin Biochem 14: 421-427.

21. Matthews DR, Hosker JP, Rudenski AS, Naylor BA, Treacher DF, et al. (1985) Homeostasis model assessment: insulin resistance and beta-cell function from fasting plasma glucose and insulin concentrations in man. Diabetologia 28: 412-419.

22. Park H, Kim K (2012) Relationship between alcohol consumption and serum lipid levels in elderly Korean men. Arch Gerontol Geriatr 55: 226-230.

23. Craig CL, Marshall AL, Sjostrom M, Bauman AE, Booth ML, et al. (2003) International physical activity questionnaire: 12-country reliability and validity. Med Sci Sports Exerc 35: 1381-1395.

24. Waters DL, Baumgartner RN (2011) Sarcopenia and obesity. Clin Geriatr Med 27: 401-421.

25. Lim JS, Lee DH, Park JY, Jin SH, Jacobs DR, Jr. (2007) A strong interaction between serum gamma-glutamyltransferase and obesity on the risk of prevalent type 2 diabetes: results from the Third National Health and Nutrition Examination Survey. Clin Chem 53: 10921098.

26. Zoppini G, Targher G, Trombetta M, Lippi G, Muggeo M (2009) Relationship of serum gamma-glutamyltransferase to atherogenic dyslipidemia and glycemic control in type 2 diabetes. Obesity (Silver Spring) 17: 370-374.

27. Hong HC, Hwang SY, Choi HY, Yoo HJ, Seo JA, et al. (2014) Relationship between sarcopenia and nonalcoholic fatty liver disease: the Korean Sarcopenic Obesity Study. Hepatology 59: 1772-1778.

28. Angulo P (2002) Nonalcoholic fatty liver disease. $N$ Engl J Med 346: 1221-1231.

29. Moylan JS, Reid MB (2007) Oxidative stress, chronic disease, and muscle wasting. Muscle Nerve 35:411-429.

30. Examination Committee of Criteria for 'Obesity Disease' in Japan; Japan Society for the Study of Obesity (2002) New criteria for 'obesity disease' in Japan. Circ J 66: 987-992.

31. Oh SW (2011) Obesity and metabolic syndrome in Korea. Diabetes Metab J 35: 561-566.

32. Chen Z, Han CK, Pan LL, Zhang HJ, Ma ZM, et al. (2014) Serum alanine aminotransferase independently correlates with intrahepatic triglyceride contents in obese subjects. Dig Dis Sci 59: 2470-2476. 